\title{
PREDICTION OF CRACKING IN RC WALLS UNDER CYCLIC LOADING BY MEANS OF A NOVEL GLOBAL CONSTITUTIVE MODEL
}

\author{
MIQUEL HUGUET ${ }^{*}$, PANAGOTIS KOTRONIS ${ }^{\dagger}$, SILVANO ERLICHER ${ }^{\dagger \dagger}$ AND \\ FRANÇOIS VOLDOIRE ${ }^{\dagger \dagger \dagger}$ \\ EGIS Industries \\ Ecole Centrale de Nantes, Université de Nantes, CNRS, \\ GeM (Institut de Recherche en Génie Civil et Mécanique), \\ Nantes, France \\ e-mail: miquel.huguet-aguilera@egis.fr \\ Ecole Centrale de Nantes, Université de Nantes, CNRS, \\ GeM (Institut de Recherche en Génie Civil et Mécanique), \\ e-mail: panagiotis.kotronis@ec-nantes.fr \\ $\dagger^{\dagger \dagger}$ EGIS Industries \\ Montreuil, France \\ e-mail: silvano.erlicher@egis.fr \\ $+1$ \\ Electricité de France R\&D \\ IMSIA UMR EDF-CNRS-CEA-ENSTA 9219 \\ Clamart, France \\ e-mail: francois.voldoire@edf.fr
}

Key words: Reinforced Concrete, Global Constitutive Model, Cyclic Loading, Cracking, Tension Stiffening

\begin{abstract}
In Reinforced Concrete (RC) structures, cracking is often a critical design parameter since engineering design codes limit the maximum crack opening to preserve the durability, tightness and aesthetics of RC buildings; robust and reliable crack opening computation methods are therefore necessary. For the case of large RC buildings (in particular for nuclear power plants), and specially for the case of cyclic (seismic) loadings, computational demanding finite element calculations are needed and so overall modeling is used. In this article, we propose to calculate the crack opening by means of a novel global (stress-resultant) nonlinear constitutive model for RC walls that incorporates crack opening as an internal variable. By means of an analytical averaging procedure and suitable physical hypotheses, four different local nonlinear phenomena are taken into account in the global model formulation [1]: (i) concrete cracking in two different crack directions and permitting both normal and tangential relative crack displacements; (ii) concrete stiffness reduction modeled by a scalar damage variable; (iii) steel-concrete slip and interface bond stresses, which are at the origin of the tension stiffening effect; and (iv) steel yielding localized at the cracks. The model is able to reproduce the behavior of RC plates submitted to in-plane and out-of-plane cyclic solicitations. Validation is provided by comparison with several experimental tests on RC structural elements, accounting for a large range of solicitations. Results show a good agreement both at global (force-displacement curves) and local (crack opening) levels.
\end{abstract}




\section{INTRODUCTION}

Crack assessment is of great importance in the design of new Reinforced Concrete (RC) structures or the evaluation of the existing ones. In particular, a good estimation of the crack opening in such structures is necessary in order to validate the specifications of civil engineering design codes concerning the permitted maximum crack opening in order to preserve the durability, tightness and aesthetics of RC buildings.

However, the common practice in nuclear civil engineering in France is to perform the structural analysis of a whole building assuming a linear elastic behavior for RC, since the global modeling approaches (models formulated in generalized stress and strains and accounting to $\mathrm{RC}$ as a single material) are used when performing Finite Element (FE) analysis, in particular when performing timeexpensive dynamic computations. Although in some specific cases more complex constitutive models are used in the global FE modeling of RC structures (see e.g. DHRC model [1] for $\mathrm{RC}$ plates), cracking is not usually explicitly taken into account in the model formulation. Therefore, a post-treatment of the computed structural stress is necessary in order to assess cracking in the structure.

In the case of $\mathrm{RC}$ beams, civil engineering codes as Eurocode 2 (EC2) [2] and Model Code 2010 (MC10) [3] the crack opening $w_{n}$ is supposed to be calculated with:

$$
w_{n}=s_{r} \frac{\sigma_{s}-\frac{k_{t} f_{c t}}{\rho_{s, e f f}}\left(1+\frac{E_{s}}{E_{c}} \rho_{s, e f f}\right)}{E_{s}}
$$

where $\sigma_{s}$ is the stress in the steel reinforcement supposing that all the tensile effort calculated in the $\mathrm{FE}$ analysis passes through it, $k_{t}$ is the tension stiffening coefficient, $f_{c t}$ is the concrete tensile strength, $E_{s}$ and $E_{c}$ are the steel and concrete Young's modulus respectively, $\rho_{s, e f f}$ is the reinforcement ratio accounting for the concrete section submitted to tension and $s_{r}$ is the crack spacing. When calculating the maximum crack opening in order to verify the stipulated authorized crack opening values in the RC structure (depending on the chemical aggressiveness of the site regarding steel corrosion), both codes EC2 and MC10 indicate that the associated maximum crack spacing $s_{\text {ro }}$ is calculated as:

$$
s_{r o}=K_{1} \cdot c+K_{2} \cdot \phi / \rho_{s, e f f}
$$

For example, in the case of concrete beams reinforced with ribbed bars and submitted to pure tensile short term loadings, the coefficients are $K_{1}=3.4$ and $K_{2}=0.34$ for EC2 and $K_{1}=2$ and $K_{2}=0.277$ for MC10.

Civil engineering codes give less information about how computing crack openings in two-dimensional RC elements, as $\mathrm{RC}$ walls or slabs, as a post-treatment of the stress previously computed in the structural FE analysis. Nevertheless, as shown in [4] for the case of cracking in RC walls, the obtained results highly depend on the post-treatment approach (a number can be found in the literature) and on the constitutive model used for the structural analysis.

Therefore, the FE analysis with a RC constitutive model adapted to global modeling and where cracking is explicitly taken into account is a suitable solution to calculate crack openings in two-dimensional RC elements. Since the mechanical behavior and cracking are coupled in the FE computations, this solution ensures a better estimation of both global structural response and crack opening evolution.

The description of cracking at the global scale in RC walls is analyzed in section 2 . This nonlinear phenomenon is one of the main nonlinearities of the behavior of the global constitutive model presented in section 3 . The capacities of the model to predict cracking as a result of a FE analysis able to reproduce the nonlinear behavior of RC structures are shown by comparison with experimental results: a RC beam submitted to pure tension and a $\mathrm{RC}$ wall submitted to a non-reversed cyclic shear loading are analyzed in sections 4 and 5, respectively. 


\section{CRACKING IN RC WALLS}

\subsection{Experimental observations}

The crack pattern in a RC structure evolves with the applied loading. Figure 1 shows the typical evolution of the crack pattern in a RC wall (with orthotropic reinforcement, where the horizontal and vertical layers of steel bars define the $x$ and $y$ axes, respectively) when it is submitted to an increasing monotonic horizontal load creating a quite uniform shear stress state. Figure 1 (c) represents the stabilized cracking state of the wall for the considered loading: an increase of the force magnitude does not create any new crack but rather an increase of the openings of the existing ones. At this state, the crack pattern associated to a quite uniform stress state in the wall is quite regular. Therefore, a group of cracks with a similar average crack orientation $-\pi / 2<\theta_{r} \leq \pi / 2$ (measured clockwise from $x$ axis) and average spacing $s_{r}$ is defined as a family of cracks.
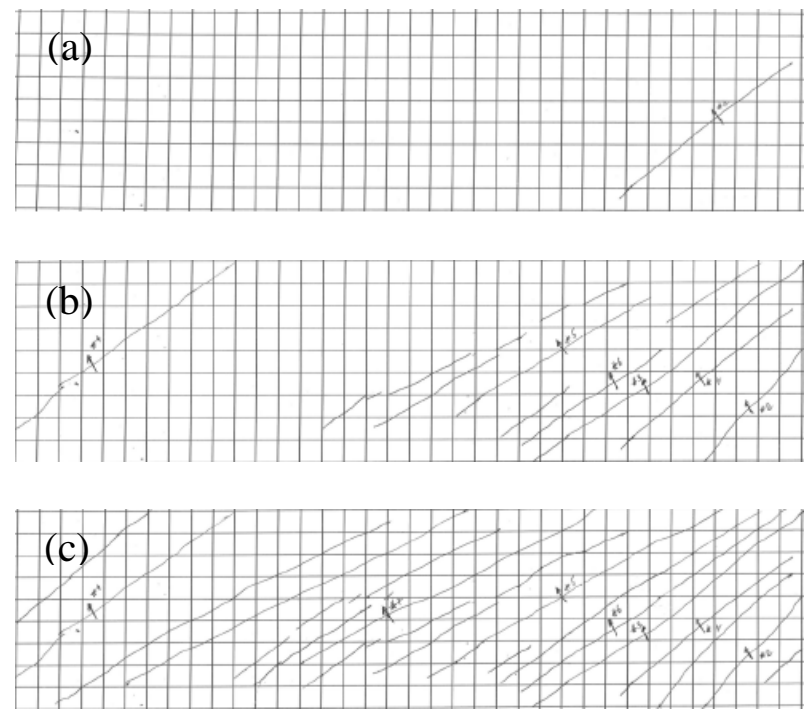

Figure 1: Crack pattern of the RC wall 3 of the CEOS.fr project [5] at (a) $900 \mathrm{kN}$, (b) $2400 \mathrm{kN}$ and (c) $4200 \mathrm{kN}$ load levels

When a RC wall is submitted to a cyclic loading, other families of cracks (related to other states of stress) can be developed. In a $\mathrm{RC}$ wall submitted to horizontal reversed cyclic loading (corresponding to a typical seismic action), a second family of cracks appears, which is also characterized by quite constant crack orientation $\theta_{r 2}$ and spacing $s_{r 2}$, see Figure 2.

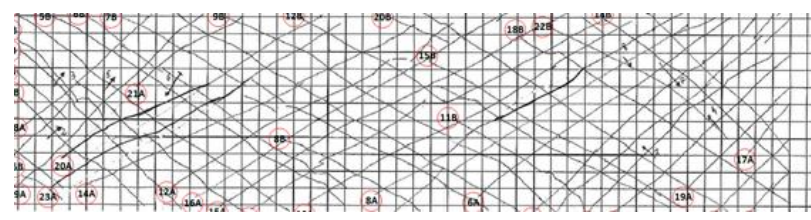

Figure 2: Crack pattern of the RC wall 2 of CEOS.fr [6]

\subsection{Cracking in the developed model}

The model developed in the present work follows a "fixed crack approach", so crack orientation $\theta_{r}$ and spacing $s_{r}$ remain constant after crack onset. This implies that the model only considers two states regarding the cracking development: the uncracked and the stabilized cracking states. Crack onset is supposed to take place when the classical Rankine criterion is reached by concrete stress $\boldsymbol{\sigma}^{c}$ (bold type is used to represent second order tensors) in tension (corresponding to the concrete tensile strength $f_{c t}$ ):

$$
F_{c r}\left(\boldsymbol{\sigma}^{c}\right)=\sigma_{1}^{c}-f_{c t} \leq 0
$$

where $\sigma_{1}^{c}$ is the first principal concrete stress. At this moment, crack is supposed to form perpendicularly with respect to the maximum tensile stress direction:

$$
\theta_{r}=-\frac{1}{2} \operatorname{atan} \frac{2 \sigma_{x y}^{c}}{\sigma_{y y}^{c}-\sigma_{x x}^{c}}
$$

Crack spacing, which is defined in the direction orthogonal to cracks, is then calculated with the formula given by Vecchio and Collins [7]:

$$
s_{r}=\left(\frac{\sin \left|\theta_{r}\right|}{s_{r x}}+\frac{\cos \theta_{r}}{s_{r y}}\right)^{-1}
$$

where $s_{r x}$ and $s_{r y}$ are the crack spacings in the equivalent RC tie-beams in the $x$ and $y$ directions respectively. $s_{r x}$ and $s_{r y}$ values can be calculated for example with the formulas given by the design codes (2), considering that they account for the maximum spacing $s_{r o}$, which is supposed to be 1.7 times the average crack spacing $s_{r}$.

The onset of a second family of cracks is governed by the same Rankine criterion (3), 
where the concrete stresses are influenced by the presence of previous cracks. Thus, the tensile strength will not be attained in directions near to the perpendicular of the existing crack: its presence, limiting the tensile stress to $f_{c t}$ in this direction, prevents new families of cracks of appearing in a close direction in relation of the first one. Consequently, and with the objective of keeping a relatively simplicity, it is decided that the model can account for a maximum of 2 families of cracks (see Figure 3), since it is considered that different cracks have to be separated by more than $60^{\circ}$ :

$$
\left|\theta_{r 1}-\theta_{r 2}\right|>60^{\circ}
$$

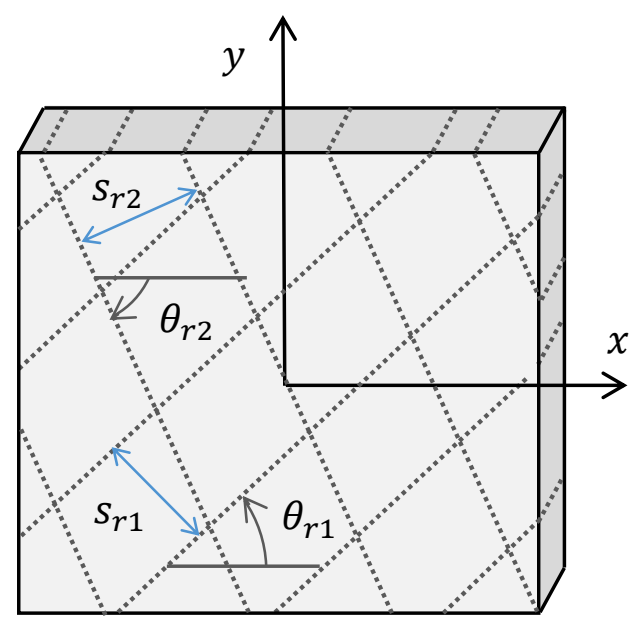

Figure 3: Geometry of the considered cracked RC wall

\section{GLOBAL CONSTITUTIVE MODEL FOR RC MEMBRANES}

The model formulated in the present paper aims at representing the nonlinear behaviour of RC membranes submitted to cyclic loadings. For doing this, four different physical phenomena (considered as the main nonlinear mechanisms affecting the RC mechanical behaviour) are taken into account: concrete cracking, concrete damage, steel-concrete slip and steel yielding.

\subsection{Concrete cracking}

Concrete displacement at cracks is defined by its normal $w_{n}$ and tangential $w_{t}$ components with respect to the cracks, which are assembled in the internal variable $w=$ $\left(w_{n}, w_{t}\right)$. In despite of the displacement discontinuity, the aggregates at cracks surface imply the presence of concrete stresses at cracks. In the present model, the bridging and the aggregate interlock stresses are retained for the description of normal and tangential concrete stresses at cracks, respectively.

The retained law for the bridging stress (or post-peak tensile concrete behavior) has a linear softening slope envelope $G_{n}$ assuring an energy dissipation equal to the concrete fracture energy $G_{f}=\int_{0}^{+\infty} G_{n}\left(w_{n}\right) d w_{n}$ :

$$
G_{n}\left(w_{n}\right)=f_{c t}-\frac{f_{c t}^{2}}{2 G_{f}} w_{n} \geq 0
$$

As seen in Figure 4, unloading is done at constant crack opening until reaching compressive stresses, where a fraction of $\alpha_{u}$ of the initial concrete stiffness is found (red-color slope). Total recovery of concrete stiffness is obtained at the crack reclosure, which is supposed to take place at $\alpha_{r} w_{n}^{\max }$, where $w_{n}^{\max }$ is the maximum historical crack opening.

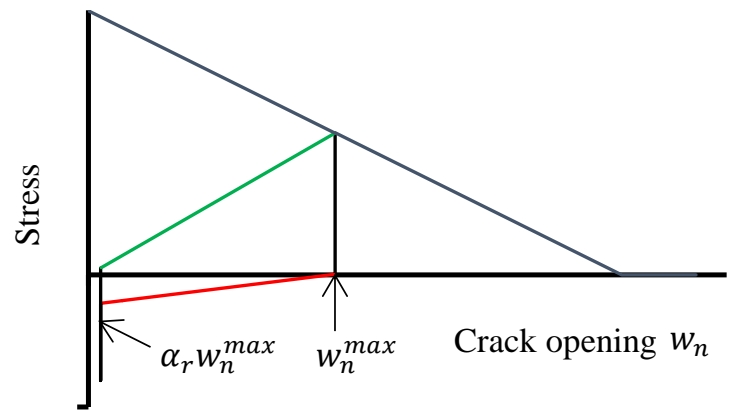

Figure 4: Retained bridging stress law, blue = loading, red $=$ unloading, green $=$ reloading

The tangential displacement at cracks is resisted by the aggregate interlock in concrete and the dowel effect in reinforcement. The latter effect and the dilatancy of concrete cracks due to the tangential displacement are not taken into account in the model. The aggregate interlock stress in concrete is modeled with the cyclic law of Figure 5. 


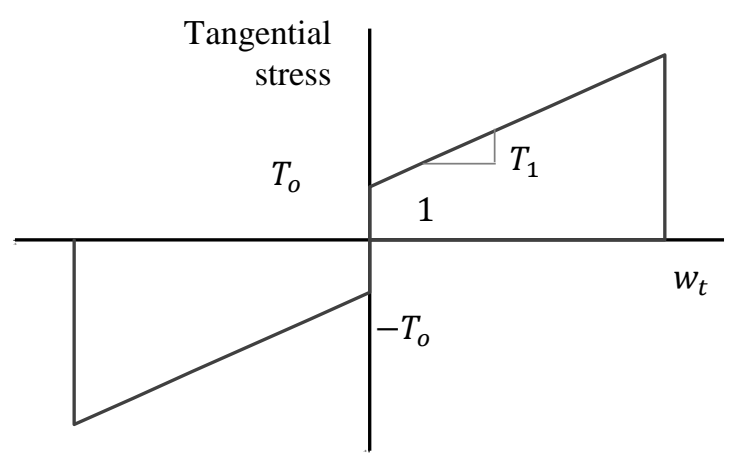

Figure 5: Retained aggregate interlock law

\subsection{Concrete damage}

The onset and development of homogeneous diffuse micro-cracking in concrete results in a stiffness reduction. An isotropic concrete damage is considered to represent this phenomenon in the formulation of the present model:

$$
\boldsymbol{\sigma}^{c}=\zeta(d) \mathbb{C}_{c}: \boldsymbol{\varepsilon}^{c}=\frac{1+\gamma_{d} d}{1+d} \mathbb{C}_{c}: \boldsymbol{\varepsilon}^{c}
$$

where $\mathbb{C}_{c}$ is the concrete elasticity tensor and $\zeta(d)$ is the damage function, which depends on the damage variable $d$ and on the $\gamma_{d}$ parameter. A constant threshold for the energy release rate is adopted and in consequence $\gamma_{d}$ is the ratio of the concrete tangent stiffness in the damage evolution phase over the initial stiffness

\subsection{Steel-concrete bond slip}

The concrete displacement discontinuity due to cracking implies a relative slip $s$ with respect to the reinforcement bars, generating bond stresses $\tau$ at the interface between the two materials. A local linear bond-slip law of local stiffness $K_{l}$ is retained $\tau=-K_{l} s$. The model being formulated at the global scale, it is defined the global bond-slip law for the average bond $\underline{\tau}^{o}$

$$
\underline{\tau}^{o}=\boldsymbol{K}_{t} \cdot\left(\boldsymbol{M}^{v w} \cdot \underline{w}-\underline{v}^{p}\right)
$$

where the global bond-slip stiffness tensor $\boldsymbol{K}_{t}$ is calculated from $K_{l}, \boldsymbol{M}^{v w}$ is the geometrical matrix transforming the internal variable crack displacement into steel-concrete slip at cracks, and $\underline{v}^{p}$ is the inelastic slip internal variable, which is included in the model in order to define a elastic-plastic law which limits the average tension stiffening in concrete to $k_{t} f_{c t}$, as indicated in the civil engineering codes EC2 and MC10 (with a value of $k_{t}=0.6$ for short term monotonic loadings). The tension stiffening effect is the direct consequence of the bond stress transmission from steel to concrete between two consecutive cracks, see Figure 6. Thus, in monotonic loadings, the structural response of the RC element is stiffer than the response of the reinforcement bars without the concrete contribution. When unloading in a cyclic load history, this effect reverses its sign and the response becomes less stiff; this negative tension stiffening phenomenon is well represented by the retained elastic-plastic law with a constant threshold of $k_{t} f_{c t}$ both loading and unloading.

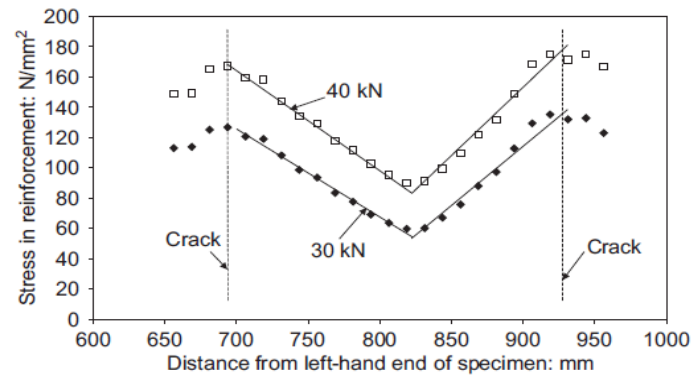

Figure 6: Measured stress in reinforcement in a cracked $\mathrm{RC}$ beam [8]

\subsection{Steel yielding}

Steel reinforcement bars can yield in a RC wall submitted to important loadings. The maximum steel concrete stress is located at cracks due to the tension stiffening effect, so the onset of steel yielding occurs at this section.

A perfectly elastic-plastic law is retained for modeling the steel reinforcement yielding at cracks, in order to limit the maximum steel stress to its yielding stress $f_{s y}$. Therefore, with this assumption yielding is only concentrated at cracks and the behavior of steel bars between them is considered as linear elastic. The internal variable $\underline{\varepsilon}^{p s}=\left(\varepsilon_{x}^{p s}, \varepsilon_{y}^{p s}\right)$ is introduced to describe the plastic steel strains of $x$ and $y$ reinforcement. 


\subsection{Analytical multi-scale analysis}

In the previous sections, the considered four nonlinear mechanisms are analyzed and formulated at the local scale. An analytical multi-scale analysis has been performed in order to insert them in the formulation of the global model for RC membrane. In particular, the generalized stress $\boldsymbol{N}$ - generalized strain $\boldsymbol{\epsilon}$ relationship is first established for a $\mathrm{RC}$ wall with one family of cracks and then the result is generalized to the case of a second family of cracks.

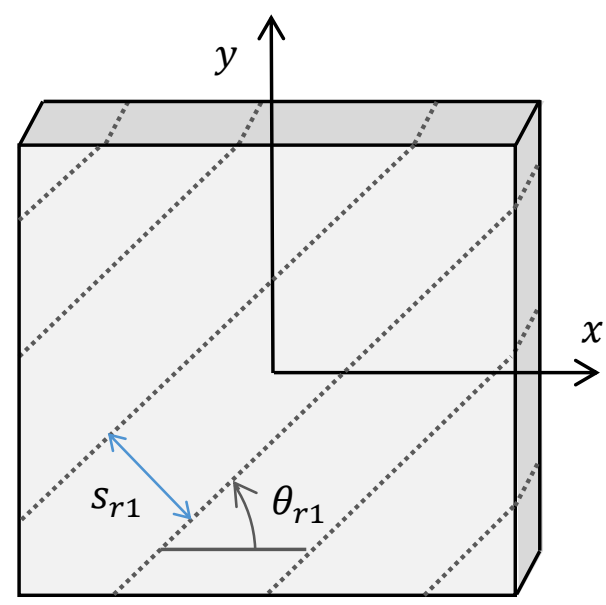

Figure 7: RC wall with one family of cracks

Figure 7 shows that a RC membrane with one family of cracks can be considered as a succession of RC struts separated by consecutive cracks. Since the width $s_{r}$ of the struts is much smaller than their length (of the order of the element dimension), the RC wall can be seen as homogeneous in the direction parallel to cracks and all the heterogeneities, which are only caused by cracks, are defined in the direction perpendicular to the crack orientation. Therefore, strain and stress local strain fields of concrete and $x$ and $y$ reinforcement bars are only dependent on the latter direction. Under this assumption, the following two-scale procedure for the establishment of the $\boldsymbol{N}-\boldsymbol{\epsilon}$ can be performed analytically between two consecutive cracks:

- Localization of the generalized membrane stress $\boldsymbol{N}$ for the identification of the local stress fields $\boldsymbol{\sigma}^{c}, \boldsymbol{\sigma}^{s x}$ and $\boldsymbol{\sigma}^{s y}$ for concrete and steel reinforcement in the $x$ and $y$ directions.

- Application of the local constitutive laws for concrete (8) and steel reinforcement (linear elastic assumption) for obtaining the local strain fields $\boldsymbol{\varepsilon}^{c}, \boldsymbol{\varepsilon}^{s x}$ and $\boldsymbol{\varepsilon}^{s y}$.

- Definition of the compatibility between the averages of local strain fields in function of the generalized strain $\boldsymbol{\epsilon}$ :

$$
\left\{\begin{array}{c}
\epsilon_{x x}=\left\langle\varepsilon_{x x}^{s x}\right\rangle+\varepsilon_{x}^{p s} \\
\epsilon_{y y}=\left\langle\varepsilon_{y y}^{s y}\right\rangle+\varepsilon_{y}^{p s} \\
\boldsymbol{\epsilon}=\left\langle\boldsymbol{\varepsilon}^{c}\right\rangle+\boldsymbol{\varepsilon}^{r}
\end{array}\right.
$$

where $\quad \boldsymbol{\varepsilon}^{r}=1 / s_{r} \underline{w} \otimes^{s} \underline{n} \quad$ is the crack equivalent strain, with $\underline{n}$ the unit normal vector with respect to crack surface.

The obtained solution for the generalized stress is [9]:

$$
\boldsymbol{N}=\mathbb{A}(d): \boldsymbol{\epsilon}-\mathbb{B}(d) \cdot \underline{w}-\mathbb{C} \cdot \underline{\varepsilon}^{p s}
$$

where $\mathbb{A}(d)$ is the fourth order elastic tensor and $\mathbb{B}$ (which depends on $\theta_{r}$ and $s_{r}$ ) and $\mathbb{C}$ are the third order tensors describing the effect on the generalized stress of crack displacement and steel plastic strain, respectively.

A second solution of the performed analysis is the expression of the concrete stress at cracks $\underline{g}$, which is the normal $n n$ and tangential $t n$ projections of the difference between the average concrete stress and the average tension stiffening stress in concrete $\boldsymbol{\sigma}^{\tau}$ :

$$
\underline{g}=\left[\mathbb{C}_{c}(d):\left(\boldsymbol{\epsilon}-\boldsymbol{\varepsilon}^{r}\right)-\boldsymbol{\sigma}^{\tau}\right]_{n n, t n}
$$

The tension stiffening stress is proportional to the global bond stress $\tau^{o}$; therefore, under the retained assumption (9), it is proportional to the steel-concrete slip $\left(\boldsymbol{M}^{v w} \cdot \underline{w}-\underline{v}^{p}\right)$.

\subsection{Formulation of the model in the Thermodynamics of Irreversible Processes framework}

The associated Helmholtz free energy surface density of the RC membrane with one family of cracks is defined as the sum of the energies associated to the generalized stress $\boldsymbol{N}$ and to the average tension stiffening stress in concrete $\boldsymbol{\sigma}^{\tau}$. 
Then, in order to generalize the obtained result to the case of a membrane with two families of cracks, it is assumed that the effects of both crack displacements on the generalized stress (11) and on concrete stress at cracks (12) are the sum of the individual effects.

The resulting Helmholtz free energy surface density of the modeled RC membrane, which depends on the observable membrane strain $\boldsymbol{\epsilon}$ variable and on the internal variables $\underline{w}_{1}, \underline{w}_{2}, \underline{v}^{p}, \underline{\varepsilon}^{p s}$ and $d$, can be expressed in the following form:

$\psi^{o}\left(\boldsymbol{\epsilon}, \underline{w}_{1}, \underline{w}_{2}, \underline{v}^{p}, \underline{\varepsilon}^{p s}, d\right)=$ $1 / 2 \boldsymbol{\epsilon}: \mathbb{A}(d): \boldsymbol{\epsilon}-\boldsymbol{\epsilon}: \mathbb{B}_{\beta}(d) \cdot \underline{w}_{\beta}$

$-\boldsymbol{\epsilon}: \mathbb{C} \cdot \underline{\varepsilon}^{p s}+1 / 2 \underline{w}_{\beta} \cdot \boldsymbol{D}_{\beta}(d) \cdot \underline{w}_{\beta}$

$+1 / 2 \underline{v}^{p} \cdot \boldsymbol{E}(d) \cdot \underline{v}^{p}+1 / 2 \underline{\varepsilon}^{p s} \cdot \boldsymbol{F} \cdot \underline{\varepsilon}^{p s}$

$-\underline{w}_{\beta} \cdot \boldsymbol{G}_{\beta}(d) \cdot \underline{v}^{p}+\underline{w}_{1} \cdot \boldsymbol{H}(d) \cdot \underline{w}_{2}$

where $\mathbb{B}_{\beta}$ is the third order tensor describing the effect of crack $\beta=1,2$ displacements on the generalized stress; and $\boldsymbol{D}_{\beta}(d), \boldsymbol{E}(d), \boldsymbol{F}$, $\boldsymbol{G}_{\beta}(d)$ and $\boldsymbol{H}(d)$ are the second order tensors describing the coupling between the internal variables.

The dissipative thermodynamic forces of the model are calculated from the derivation of the Helmholtz free energy surface density (13) with respect to the internal variables:

$$
\begin{array}{ll}
Y=-\frac{\partial \psi^{o}}{\partial d} & \underline{q}_{r \beta}=-\frac{\partial \psi^{o}}{\partial \underline{w}_{\beta}} \\
\underline{q}_{v}=-\frac{\partial \psi^{o}}{\partial \underline{v}^{p}} & \underline{q}_{s}=-\frac{\partial \psi^{o}}{\partial \underline{\varepsilon}^{p s}}
\end{array}
$$

In particular, it is verified that the constitutive law (11) is obtained by the derivation of (13) with respect to the generalized strain $\boldsymbol{\epsilon}$.

Finally, the flow of the internal variables is defined by the normality rule with respect of the associated threshold functions $f_{g, n}, f_{g, t}, f_{d}$, $f_{v}$ and $f_{s}$, which correspond to the local constitutive laws defined previously for the four considered nonlinear physical phenomena at the local scale affecting the global behavior of a $\mathrm{RC}$ wall $(\alpha=x, y)$ : $\dot{d}=\dot{\lambda}_{d} \frac{\partial f_{d}}{\partial Y}$

$\dot{w}_{\beta, n}=\dot{\lambda}_{g \beta, t} \frac{\partial f_{g \beta, t}}{\partial q_{r \beta, n}}$

$\dot{w}_{\beta, t}=\dot{\lambda}_{g \beta, t} \frac{\partial f_{g \beta, t}}{\partial q_{r \beta, t}}$

$\dot{v}_{\alpha}^{p}=\dot{\lambda}_{v, \alpha} \frac{\partial f_{v, \alpha}}{\partial q_{v, \alpha}} \quad \dot{\varepsilon}_{\alpha}^{p s}=\dot{\lambda}_{s, \alpha} \frac{\partial f_{s, \alpha}}{\partial q_{s, \alpha}}$

More details about the model formulation in the framework of Thermodynamics of Irreversible Processes are given in [10].

\subsection{Extension of the model to RC plates submitted to out-of-plane loading}

The extension of the model to RC plates submitted to both membrane loadings and outof-plane bending moments is obtained by supposing that the RC plate can be discretized in its height in four (two RC and two pure concrete C) membranes, as illustrated in Figure 8.

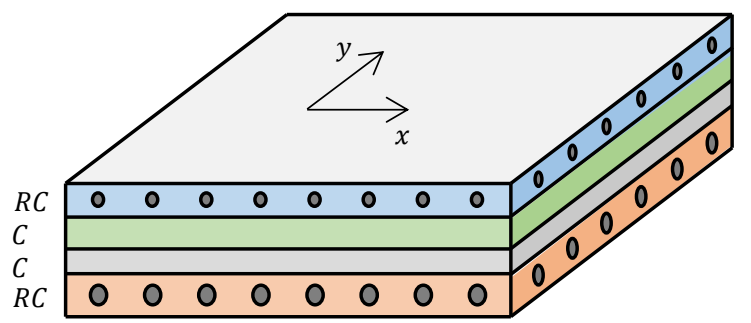

Figure 8: Discretization of a RC plate section for the generalization of the model to out-of-plane solicitations

Internal variables $\underline{w}_{1}, \underline{w}_{2}, \underline{v}^{p}, \underline{\varepsilon}^{p s}$ and $d$ are supposed to be different (and constant) in each of the two halves of the height section, taking into account that $\underline{v}^{p}$ and $\underline{\varepsilon}^{p s}$ are not defined in the pure concrete sections. Under these assumptions, the total Helmholtz free energy surface density of the RC plate is obtained as the sum of the free energies of each of the discretized layers, given by the result found in (13). The internal variables flows are defined for each of the two halves of the plate equivalently to (15). For the sake of simplicity, the details of this model formulation are not given here but they can be found in [10]. 


\section{APPLICATION TO A PURE TENSION TEST IN A RC TIE-BEAM}

The test N10-14 of the Farra and Jaccoud [11] experimental campaign on RC tie-beams submitted to monotonic pure tension loading is considered for the validation of the model for global force-displacement response and crack opening prediction.

The test is repeated twice in two theoretical identical RC beams of $1.15 \mathrm{~m}$ length and $100 \times 100 \mathrm{~mm}^{2}$ section made by a concrete characterized by a Young's modulus $E_{c}=$ 29.9GPa, a tensile strength $f_{c t}=1.73 \mathrm{MPa}$ (measured value in the RC beam considering the size effect and the restrained concrete shrinkage, opposed to the nominal strength of $f_{c t}=2.13 \mathrm{MPa}$ ) and a compressive strength $f_{c}=29.9 \mathrm{MPa}$. The reinforcement consists in an single bar of $\phi=14 \mathrm{~mm}$ located at the center of the section $\left(\rho_{s}=1.54 \%\right)$, characterized by a Young's modulus $E_{s}=$ $198 \mathrm{GPa}$ and a yield strength of $f_{s y}=$ $569 M P a$.

The tension stiffening coefficient is set to $k_{t}=0.6$ (following EC2 and MC10) and the local bond-slip stiffness to $K_{l}=10^{11} \mathrm{~Pa} / \mathrm{m}$. The theoretical fracture energy $G_{f}=135 \mathrm{~N} / \mathrm{m}$ calculated with $\mathrm{MC10}$ is reduced to $G_{f}=$ $88 \mathrm{~N} / \mathrm{m}$ in order to be coherent with the $f_{c t}$ diminution from its nominal to its measured value, and thus maintain the concrete tensile post-peak slope given by (7), which depends on the ratio $f_{c t}^{2} / G_{f}$.

The theoretical crack spacing in the direction of the beam axis is set equal to the one measured in the test $s_{r}=0.173 \mathrm{~m}$, in order to verify the model performances considering its formulation without adding any error due to this input parameter.

Parameters describing damage, aggregate interlock effect and unloading of the bridging stress law are not specified since the test consists in a monotonic pure tension loading and they do not affect the result.

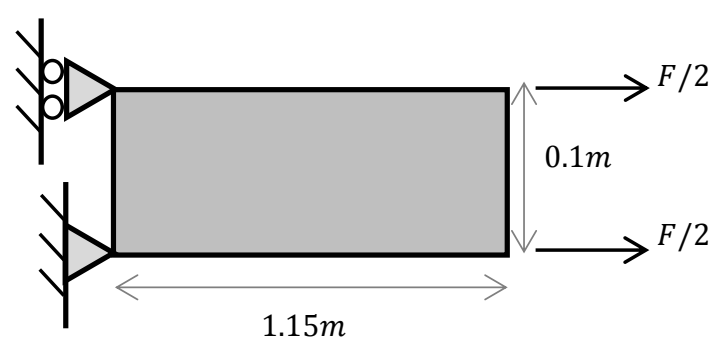

Figure 9: Model of the RC beam N10-14 of [11] with one quadrangular $\mathrm{FE}$

The RC beam is modeled with an only quadrangular DKQG (Discrete Kirchhoff Quadrangle Generalized) finite element (shell element with four Gauss integration points) in Code_Aster FE software in order to show the global modeling capacities of the model (see Figure 9).

A good agreement is observed in Figure 10 for both force-displacement (Figure 10a) and total crack opening (sum of all existing cracks in the RC beam) evolution (Figure 10c), while an underestimation of the average individual crack opening (Figure 10b) is observed at the crack formation stage.

This result is expected since in the model the element reaches the stabilized crack stage from the onset of the first crack and the crack spacing stay constant (fixed crack approach) and equal to the final average crack spacing (relatively small), while the crack pattern evolves in the experimental tests (converging to the final stabilized crack stage). Therefore, at the onset of the first crack, the crack spacing is much larger than the average final one and thus, the measured crack openings are also larger.

Nevertheless, since the generalized stressstrain relationship is based on average (computed from the total) crack openings, the total force-displacement curve is well represented in all the stages. Moreover, the maximum crack opening verification of design codes is done under the assumption of stabilized cracking, in which, as observed in this comparison with experimental results, crack openings are well estimated. 
(a)

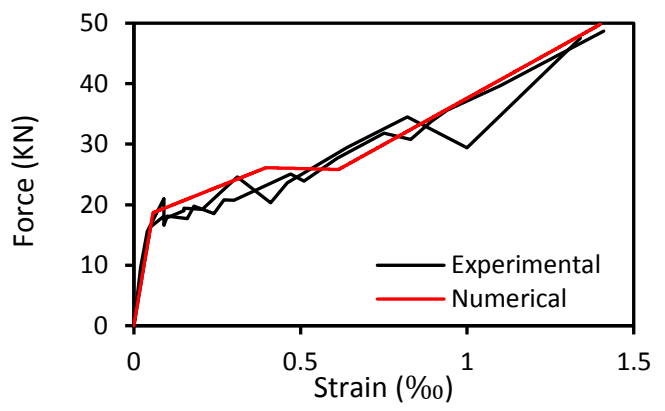

(b)

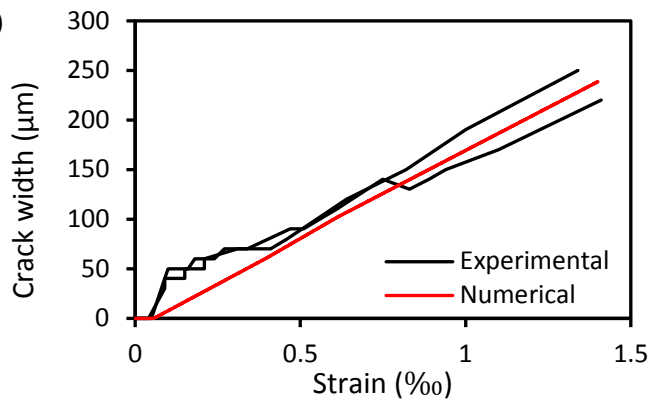

(c)

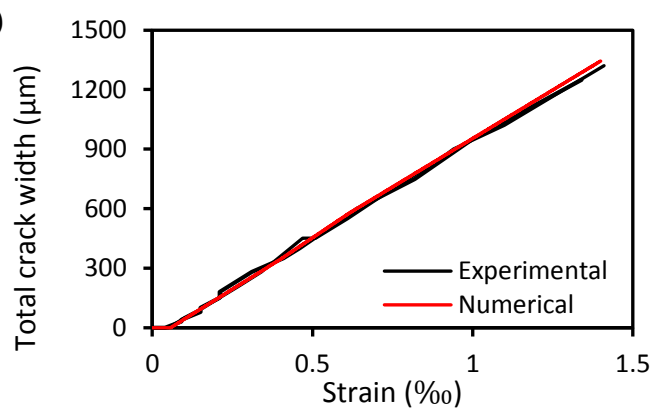

Figure 10: Experimental vs. numerical (a) forcedisplacement curve, (b) average individual crack and (c) total crack opening in RC beam N10-14 of [11]

\section{APPLICATION TO A RC WALL SUBMITTED TO CYCLIC SHEAR}

\subsection{Experimental test description}

In this section it is considered the experimental test on the RC wall number 3 [5] designed and tested in the framework of the French national research project CEOS.FR (Comportement et Evaluation des Ouvrages Spéciaux. Fissuration - Retrait / Behavior and Assessment of Special Structures. Cracking Shrinkage).

The tested specimen is a mock-up of $4200 \mathrm{~mm}$ of length, $1050 \mathrm{~mm}$ of height and $150 \mathrm{~mm}$ of thickness, with an assumed geometrical scale factor of $1 / 3$, in order to represent a standard $\mathrm{RC}$ wall used in nuclear facilities. A low 1/4 slenderness ratio and the vertical rebars of $25 \mathrm{~mm}$ and $32 \mathrm{~mm}$ diameter at the extremities prevent cracking due to global bending in the wall. The redistribution of the shear effort in the wall is assured by two horizontal highly reinforced concrete beams connected to its upper and bottom edges. In order to extend the crack formation phase in the wall before complete failure, the nonbrittleness is assured by a $1.05 \%$ reinforcement ratio.

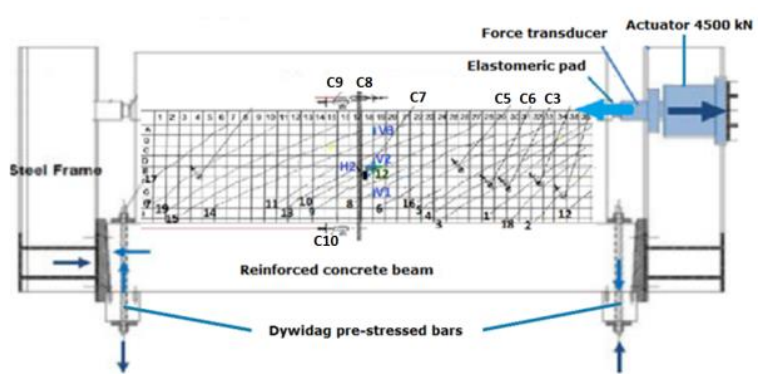

Figure 11: Scheme of the RC wall 3 of CEOS.fr - steel frame structure and its instrumentation

Figure 11 shows the application of the horizontal force by an actuator at one side of the upper beam and the interaction between the RC wall and the steel frame containing it. The applied load history is cyclic without inversion of the force sign (with increasing peak values in each cycle), see Figure 12.

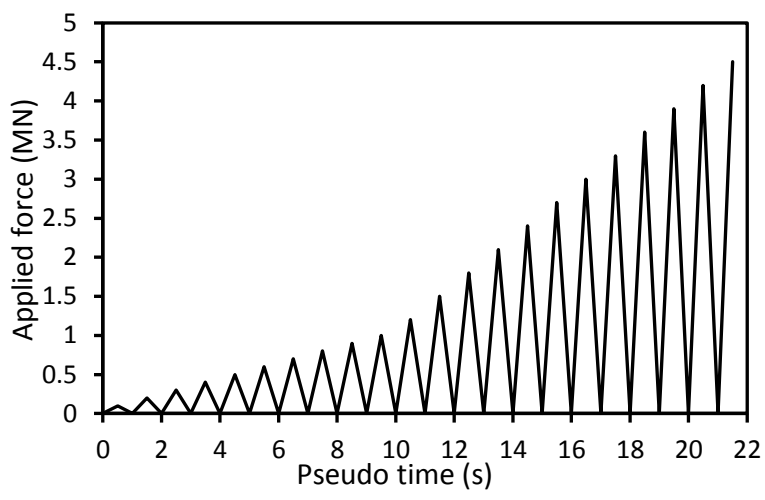

Figure 12: Applied load history on RC wall 3 of CEOS.fr

The wall is reinforced with $\phi=10 \mathrm{~mm}$ steel bars spaced by $100 \mathrm{~mm}$, characterized by Young's modulus $E_{s}=200 G P a$, mass density $7850 \mathrm{~kg} / \mathrm{m}^{3}$ and yield limit $f_{s y}=555 \mathrm{MPa}$. Concrete cover is $10 \mathrm{~mm}$ and $20 \mathrm{~mm}$ for horizontal and vertical rebars, respectively.

The experimental properties of concrete are: Young's modulus $E_{c}=27.4 G P a$, Poisson's ratio $v_{c}=0.13$, mass density 
$2200 \mathrm{~kg} / \mathrm{m}^{3}$, compression strength $f_{c}=$ 40.0MPa, tensile strength $f_{c t}=3.5 \mathrm{MPa}$ and fracture energy $G_{f}=158 \mathrm{~N} / \mathrm{m}$.

Due to the cyclic loading, a low tension stiffening coefficient $k_{t}=0.2$ is retained. The asymptotic damage slope is set to $\gamma_{d}=0.2$ and damage is supposed to appear in compression at $f_{c} / 4$, corresponding to a damage threshold $k_{o}=1459 \mathrm{~N} / \mathrm{m}$.

Concrete size effect on tensile strength is complex to be estimated in a structure with a non-uniform state of stress. Taking into account the observed and size effect in the RC beam (with smaller dimensions) of the previous section, the experimental $f_{c t}$ value is reduced by $1 / 2: f_{c t}=1.75 \mathrm{MPa}$. As previously stated, the value of the fracture energy has to be coherently and thus, a value of $G_{f}=$ $40 \mathrm{~N} / \mathrm{m}$ is used in computations.

The values of crack displacements parameters are set as: $\alpha_{r}=0.02, \alpha_{u}=0.15$, $T_{o}=1 \mathrm{MPa}$ and $T_{1}=10 \mathrm{GPa} / \mathrm{m}$.

Theoretical crack spacings in $x$ and $y$ directions are set as $s_{r x}=124 \mathrm{~mm}$ and $s_{r y}=138 \mathrm{~mm}$, calculated with the expression given in [10] as an optimization of design codes formula (2), with $K_{1}=1.37$ and $K_{2}=0.116$ as the best predictors for the average crack spacing.

\subsection{Comparison between numerical and experimental results}

The RC wall is modeled with quadrangular DKQG shell elements which have associated the new constitutive model. As seen in Figure 13 , the real boundary conditions have been represented including the steel frame, which is modeled with beam elements.

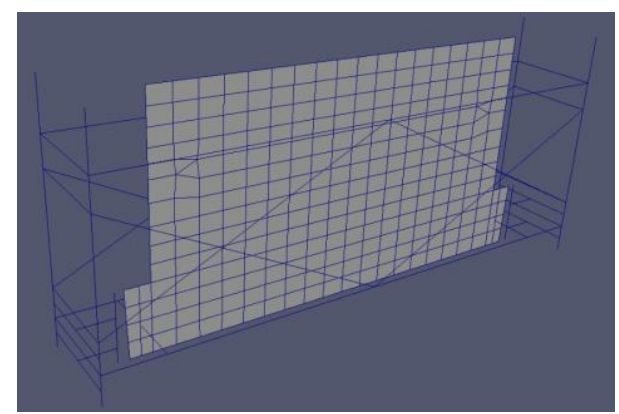

Figure 13: FE model of RC wall 3 of CEOS.fr
The first comparison concerns the curve applied force vs. relative displacement of sensors C9 and C10 (see Figure 12). Figure 14 shows that the global shear behavior is well represented, in terms of the envelope curve and also in terms of hysteretic loops in unloading-reloading cycles and the permanent displacements.

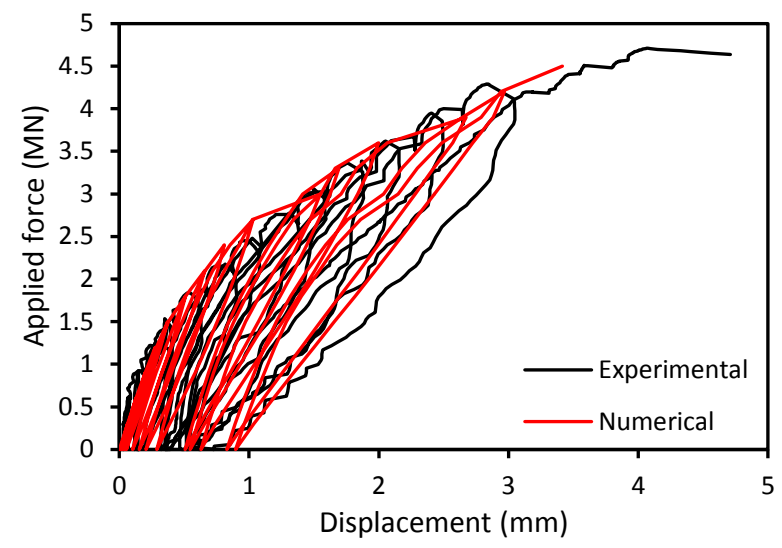

Figure 14: Experimental vs. numerical global displacement curve of RC wall 3 of CEOS.fr

The crack pattern evolution in the $\mathrm{RC}$ wall predicted by the constitutive model is compared with the experimental pattern in Figure 15 for 1500, 2400 and $3600 K N$ load levels (the latter corresponding to the experimentally observed stabilization of crack pattern). The numerical crack pattern is represented by the zones where crack opening internal variable is greater than 0 (color different from blue). The crack opening internal variable plotted in the figure is $w_{1, n}$, since the test consists in a loading-unloading with the same direction of applied force and only one principal family of cracks is generated.

The comparison shows that the constitutive model is able to predict that, under the considered applied shear loading, the first cracks appear in the lower right corner of the RC wall and then cracking propagates gradually to the left. The experimental average crack orientation of $\theta_{r} \approx 30^{\circ}$ is found in the generated crack pattern in the RC wall far from the edges. 

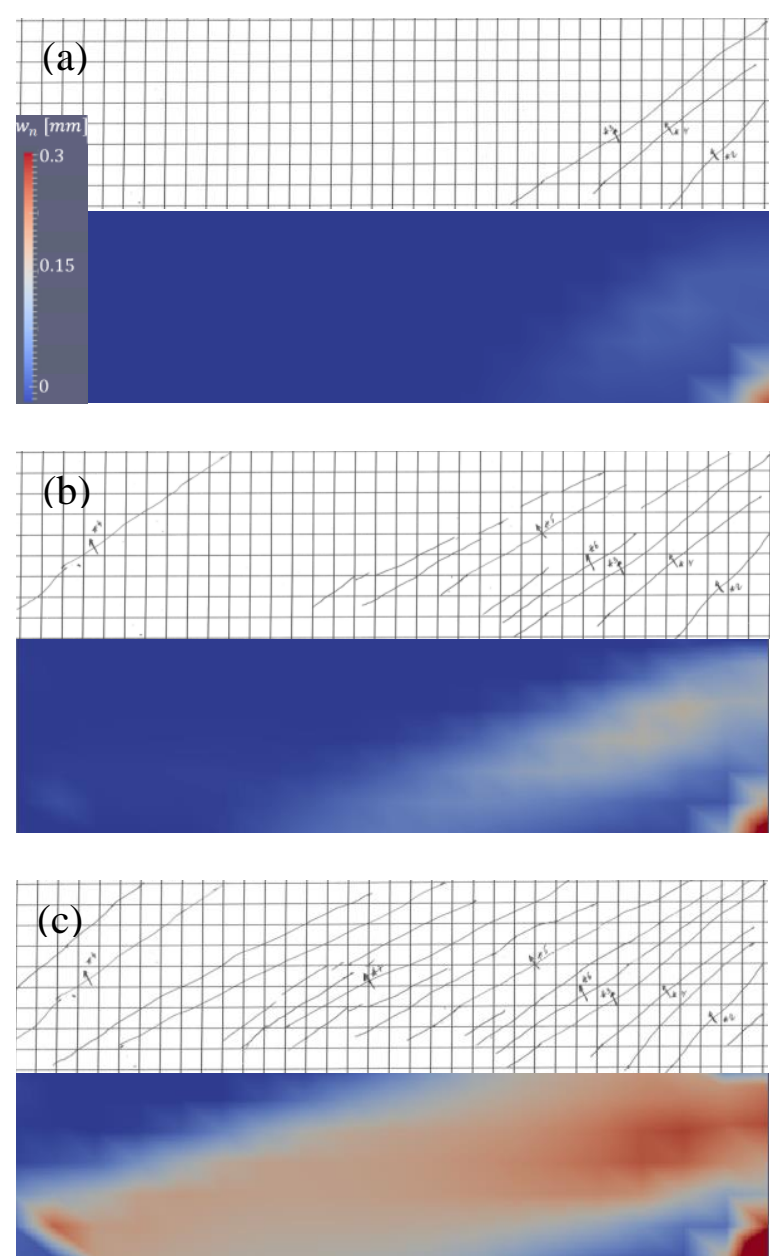

Figure 15: Experimental vs. numerical crack pattern in $\mathrm{RC}$ wall 3 of CEOS.fr for (a) $1500 \mathrm{kN}$, (b) $2400 \mathrm{kN}$ and (c) $3600 \mathrm{kN}$ load levels

A more local analysis concerning the prediction of cracking in the RC wall 3 of CEOS.fr is presented in Figure 16. The measured crack openings in LVDT sensors C3, C5, C6 and C7 (see their location on the wall in Figure 11) are compared with the computed average (maximum historical) crack opening in the FE in which each sensor is located.

The crack onset at the four sensors is well represented, in correspondence with the good fitting of the cracking evolution in Figure 15. The prediction of the crack opening evolution is satisfactory for sensors C5, C6 and C7, while the opening is underestimated by roughly a factor of 0.5 for sensor C3. It is remarked that LVDT sensors measure punctual crack opening values while the roughness of the crack surface implies that crack width has important variations at the scale of $\mathrm{mm} / \mathrm{cm}$. It is possible that sensor C3 is located at a point where the roughness of the crack makes the punctual measure much greater than the average representative value along the crack, which is the computed value with the proposed stress resultant model.
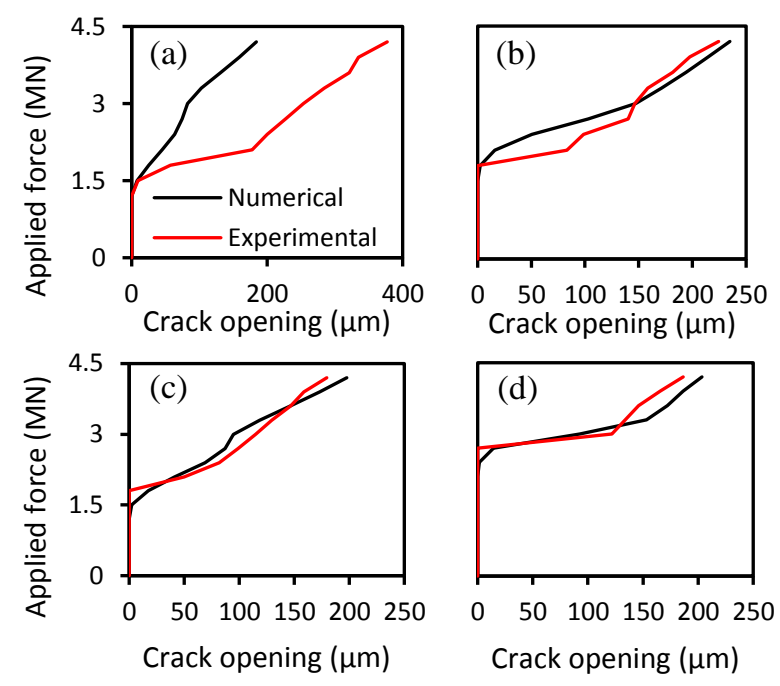

Figure 16: Experimental vs. numerical crack openings at sensors (a) C3, (b) C5, (c) C6 and (d) C7 of RC wall 3 of CEOS.fr

\section{CONCLUSIONS}

The prediction of cracking in RC walls by means of a new stress resultant constitutive model for RC walls is proposed and implemented in a global FE modeling. A fixed crack approach is retained for the description of the crack pattern (constant orientation and spacing): the stress state at cracking onset determines the crack orientation and then the crack spacing is calculated with existing formulas of the literature depending on the geometry. Two different families of cracks are considered to represent the typical crack pattern in a RC wall submitted to seismic loadings. At cracks, concrete is assumed to carry bridging (normal) stresses accounting for the crack opening-reclosing and tangential stresses due to the aggregate interlock. The bond-slip stress at the interface between concrete and reinforcement bars, which is caused by the differential slip due to cracking, is considered to reproduce the tension stiffening effect. As a consequence of this 
phenomenon, steel yielding of reinforcement bars is assumed to be concentrated at cracks. Under important compression stress, concrete damage is retained to model the concrete stiffness reduction due to microcracking. These considered nonlinear physical phenomena are formulated in order to be adapted to cyclic loadings, and they are put together in the constitutive model formulated at the global scale by an analytical multi-scale analysis.

The model is applied to a RC beam submitted to a pure tension loading and to a $\mathrm{RC}$ wall submitted to a cyclic non reversed shear loading. The comparison with the experimental results shows a good fitting to the monotonic and cyclic structural (global force-displacement curves) response, including unload-reload hysteretic cycles and permanent strains. Cracking is also well predicted: the comparison of crack opening evolution shows a good agreement, indicating that concrete stress at cracks and tension stiffening effect are well represented in the model. The crack pattern evolution in the RC wall 3 of CEOS.fr is also well estimated.

\section{REFERENCES}

[1] Combescure, C., Dumontet, H. and Voldoire F. 2013. Homogenized constitutive model coupling damage and debonding for reinforced concrete structures under cyclic solicitations. International Journal of Solids and Structures, 50:3861-3874.

[2] Comité Européen de Normalisation. 2005. EuroCode 2 - 2004 Design of concrete structures: Part 1-1.

[3] Fédération Internationale du Béton fib. 2011. Model Code 2010.

[4] Huguet, M., Eggen, T., Rachidi, M., Erlicher, S., Bou-Said, E. and Crolet, Y. 2014. Engineering method for the evaluation of seismically induced cracking in reinforced concrete nuclear buildings. Proceedings of TINCE 2014.

[5] Rivillon P. and Gabs A. 2011. Projet National de Recherche CEOS, Axe 2 Expérimentations, Rapport $n^{\circ} E E M \quad 09$
26023877- $C$, Technical Report, CSTB, (in French).

[6] Rivillon P. and Gabs A. 2011. Projet National de Recherche CEOS, Axe 2 Expérimentations, Rapport $n^{\circ}$ EEM 09 26023877-B, Technical Report, CSTB, (in French).

[7] Vecchio, F.J. and Collins, M.P. 1986. The modified compression field theory for reinforced concrete elements subjected to shear. ACI Journal, 83(2):219-231.

[8] Beeby, A.W. and Scott, R.H. 2005. Cracking and deformation of axially reinforced members subjected to pure tension. Magazine of concrete research, 57(10):611-621.

[9] Huguet, M., Voldoire, F., Kotronis, P. and Erlicher, S. 2014. Homogenized nonlinear stress resultant constitutive model for cracked reinforced concrete panels. Proceedings of 11th World Congress on Computational Mechanics; 2, pp. 13701380.

[10]Huguet, M. 2016. Homogenized stress resultant model for cracking in $R C$ plates under seismic loadings. $\mathrm{PhD}$ Thesis, Ecole Centrale Nantes / EGIS Industries.

[11]Farra, B. and Jaccoud, J.P. 1993. Influence $d u$ Béton et de l'armature sur la fissuration des structures en Béton. Rapport des essais de tirants sous déformation imposée de courte durée. Département de Génie Civil, Ecole Polytechnique Fédérale de Lausanne, Publication No.140. 